\title{
Numerous genetic loci identified for drought tolerance in the maize nested association mapping populations
}

\author{
Chunhui Li ${ }^{1}$, Baocheng Sun², Yongxiang Li', Cheng Liu², Xun Wu' ${ }^{1}$ Dengfeng Zhang ${ }^{1}$, Yunsu Shi', Yanchun Song ${ }^{1}$, \\ Edward S. Buckler ${ }^{3,4^{*}}$, Zhiwu Zhang ${ }^{5^{*}}$, Tianyu Wang ${ }^{1 *}$ and Yu Li ${ }^{1^{*}}$
}

\begin{abstract}
Background: Maize requires more water than most other crops; therefore, the water use efficiency of this crop must be improved for maize production under undesirable land and changing environmental conditions.

Results: To elucidate the genetic control of drought in maize, we evaluated approximately 5000 inbred lines from 30 linkage-association joint mapping populations under two contrasting water regimes for seven drought-related traits, including yield and anthesis-silking interval (ASI). The joint linkage analysis was conducted to identify 220 quantitative trait loci (QTLs) under well-watered conditions and 169 QTLs under water-stressed conditions. The genome-wide association analysis identified 365 single nucleotide polymorphisms (SNPs) associated with drought-related traits, and these SNPs were located in 354 candidate genes. Fifty-two of these genes showed significant differential expression in the inbred line B73 under the well-watered and water-stressed conditions. In addition, genomic predictions suggested that the moderate-density SNPs obtained through genotyping-by-sequencing were able to make accurate predictions in the nested association mapping population for drought-related traits with moderate-to-high heritability under the water-stressed conditions.
\end{abstract}

Conclusions: The results of the present study provide important information that can be used to understand the genetic basis of drought stress responses and facilitate the use of beneficial alleles for the improvement of drought tolerance in maize.

Keywords: Maize, Nested association mapping population, Drought tolerance, Joint linkage mapping, GWAS, Candidate gene

\section{Background}

Maize (Zea mays ssp. mays L.) is one of the three most important cereal crops and has the second highest cultivation area worldwide (http://faostat.fao.org). However, the productivity of this crop is frequently reduced in response to drought stress. Traditional breeding has shown limited progress in improving maize drought tolerance under water-limited conditions; therefore, determining how

\footnotetext{
*Correspondence: esb33@cornell.edu; Zhiwu.Zhang@WSU.edu; wangtianyu@caas.cn; liyu03@caas.cn

${ }^{3}$ Institute for Genomic Diversity, Cornell University, Ithaca, NY, USA

${ }^{5}$ Department of Crop and Soil Sciences, Washington State University,

Pullman, WA, USA

'Institute of Crop Science, Chinese Academy of Agricultural Sciences, Beijing

100081, China

Full list of author information is available at the end of the article
}

maize responds to drought stress will provide new tools for the genetic improvement of crop yields in arid environments [1].

Grain yield (GY) under water stress is a primary trait used to evaluate the degree of drought tolerance in maize [2]. Certain secondary traits associated with drought tolerance, such as the anthesis-silking interval (ASI), plant height $(\mathrm{PH})$ and grain yield components, are highly correlated with drought tolerance and exhibit increased heritability [3-7]. Hence, these traits have been used to improve the selection efficacy for drought tolerance in plant breeding and identify the underlying functional quantitative trait loci (QTLs)/genes that control drought tolerance [8]. 
Previous studies have reported the use of linkage analyses and/or association mapping to identify drought-related quantitative trait loci. QTL mapping for GY and agronomic traits associated with drought tolerance have been conducted in a number of different bi-parental populations under well-watered (WW) and water-stressed (WS) conditions [4, 9-12]. The QTLs for drought tolerance identified in maize are available at http://www.maizegdb.org and http://www.plantstress.com, and several researchers have collected published QTL results, and data associated with QTLs for drought stress or drought tolerance obtained in different populations were used to conduct QTL metaanalyses $[11,13-15]$ to identify consensus QTLs and shrink the QTL confidence interval. However, the QTL studies with bi-parental populations can only detect two alleles and have limited mapping power and resolution.

Furthermore, association mapping based on linkage disequilibrium has been used to identify the causal genes affecting GY and agronomic traits associated with drought responses. Lu et al. [16] identified several single nucleotide polymorphisms (SNPs) associated with ASI and $\mathrm{PH}$ under drought tolerance in 305 diverse inbred lines genotyped using a 1536 SNP array. Xue et al. [17] identified 42 drought-associated SNPs for nine agronomic traits using a 350 tropical and subtropical maize association panel and data on $56 \mathrm{~K}$ SNPs. Thirunavukkarasu et al. [18] used 240 accessions of subtropical maize and 56,110 SNPs to conduct association analyses for seven agronomic traits, including ASI, grain yield and five-grain yield component traits under WW and WS conditions, and their results indicated that 61 SNPs were significantly associated with drought tolerance. These drought studies were performed using small association panels and low marker densities that were unable to identify the global beneficial alleles for drought tolerance.

Currently, two publically available maize genetic resources called nested association mapping (NAM) populations have been developed in the US (US-NAM) and China (CN-NAM). The US-NAM population consists of 25 bi-parental families, including approximately 5000 recombinant inbred lines (RILs) [19]. The CN-NAM population consisted of $11 \mathrm{bi}$-parental families, including approximately 2000 RILs [20]. These populations provide increased mapping resources to successfully dissect the genetic architecture of different complex agronomic traits, such as the flowering time [21], leaf architecture [22], male and female inflorescences [23], kernel components traits [24], plant height [25], stalk strength [26], etc. Using these NAM populations, we systematically dissected the genetic loci controlling maize drought tolerance using a joint linkage analysis and genome-wide association studies (GWAS). In addition, both NAM populations were sequenced using the Genotyping-By-Sequencing (GBS) method, and high-density recombination maps were constructed based on the GBS-obtained data [20]. These methods improved the mapping resolution.

In the present study, two sets of NAM populations were used to determine the phenotype of seven drought-related traits under well-watered (WW) and water-stressed (WS) conditions. Joint linkage QTL mapping was performed to detect the genomic regions that control maize drought tolerance under different water regimes. GWAS was conducted to identify the candidate genes that were significantly associated with seven drought-related traits. Furthermore, the candidate genes were validated using the RNA-seq data of the inbred line B73 obtained under the WW and WS conditions. In addition, crossvalidated genomic predictions were performed to assess the accuracy of predicting drought-related traits under the two water regimes.

\section{Results}

Analysis of phenotypes under the WW and WS conditions The heritability of the seven drought-related traits and average phenotypic performance based on the BLUP values is shown in Additional file 1: Table S1 for $\mathrm{CN}$ NAM and Additional file 2: Table S2 for US-NAM. Significant differences were observed for the means of all of the traits within the two NAM populations under the WW and WS environments using the $F$ test. The results suggested that drought stress at the flowering stage had different effects on drought-related traits. The average grain yield per plant (GYPP) in the CN-NAM population decreased by $28 \%$ under WS, whereas it decreased by $66 \%$ decrease in the US-NAM population under WS. As expected, the average ASI in the two NAM populations was longer under WS than under WW because the ASI reflects the susceptibility of different genotypes to drought stress. The estimated heritability for the seven traits of the CN-NAM population under WS and WW ranged from 49.3 to $83.5 \%$ and from 61.7 to $89.4 \%$,respectively. The heritability estimates of the seven traits were all higher under WW than under WS.

The phenotypic correlations among the seven traits are listed in Additional file 3: Table S3 and Additional file 4: Table S4 for the CN-NAM and US-NAM population, respectively. Except for ASI and EL under the two water regimes, significant phenotypic correlations were observed among all the traits, and significant negative correlations were observed between the ASI and the remaining traits under both water regimes.

\section{Joint linkage mapping of drought-related traits}

We identified the QTLs that control drought tolerancerelated traits under the WW and WS conditions using a joint linkage analysis of the CN-NAM population. We identified 8-23 QTLs for the seven drought-related 
traits under WW, and they explained $23.7-66.3 \%$ of the total phenotypic variation, whereas we identified 8-20 QTLs under WS, and they explained 20.2-55.4\% of the total phenotypic variation (Table 1). A single joint QTL could explain $0.9-8.3 \%$ of the phenotypic variation under WW depending on the trait, whereas it could explain $1.2-10.4 \%$ of the phenotypic variation under WS (Additional file 5). Except for GYPP, other traits were detected as consensus QTLs under different water regimes, with a total of 35 consensus QTLs identified. The consensus QTLs for each trait could explain more than $50 \%$ of the total phenotypic variation by all QTLs detected in the corresponding trait except the KNPR. The joint linkage analysis enabled the estimation of an independent allele effect for each QTL in all $11 \mathrm{CN}$-NAM families. The total QTL allele effects ranged from 88 for GYPP to 253 for HKW under WW (31-73\% alleles were significant at $P<0.05$ ), whereas the number ranged from 88 for GYPP to 220 for PH under WS (27-43\% alleles were significant at $P<0.05$ ).

To validate the QTLs identified in the CN-NAM population, we also conducted a joint linkage analysis in the US-NAM population (Additional file 6). A total of 112 and 78 joint QTLs were detected for all of the traits in the US-NAM population under the WW and WS conditions, respectively. Among the 112 and 78 QTLs, 32 and 18 QTLs were identified under the WW and WS conditions in the CN-NAM population, respectively, and each trait under the different water conditions had consensus QTLs between the CN-NAM and US-NAM populations except for KNPR under WS (Additional file 7: Table S5).

\section{GWAS of drought-related traits}

The genome-wide association study (GWAS) identified 1075 SNPs under WW and 795 SNPs under WS with a bootstrap posterior probability (BPP) $\geq 0.05$ for all of the traits in the CN-NAM population (Additional file 8), and it identified 700 SNPs under WW and 448 SNPs under WS in the US-NAM population (Additional file 9). In certain cases, clusters of associated SNPs were detected within less than $100 \mathrm{~kb}$ of each other. The SNPs within a cluster were all identified based on a strong linkage with the same causative genes. Therefore, $100 \mathrm{~kb}$ was selected as a non-overlapping sliding window, and the SNPs with the highest statistical significance were selected as representatives of this window. A total of 778 SNPs under WW and 649 SNPs under WS for the CN-NAM population and 586 SNPs under WW and 359 SNPs under WS for the US-NAM population were identified in this manner (Fig. 1).

To identify the most robust associated SNPs, we further selected a BPP $\geq 0.25$ as a significance threshold, which detected a total of 255 and 146 strongly associated SNPs for CN-NAM and US-NAM, respectively (Fig. 1). The GWAS results for both NAM populations were compared with both NAM joint QTL intervals. In the WW treatment, $27 \%$ of the strongly associated SNPs identified in both NAM populations overlapped with both NAM joint QTL intervals, whereas in the WS treatment, 29 \% strongly associated SNPs overlapped. A total of 221 and 179 strongly associated SNPs were detected in both NAM populations under the WW and WS conditions, respectively. Among the SNPs detected under the different water regimes, 18 associated SNPs were detected in both water regimes, which suggests that these SNPs were constitutive loci in different water environments. Ultimately, 365 strongly associated SNPs were used to identify the candidate genes associated with drought tolerance.

\section{Identification of candidate genes}

The predicted genes close to each of the 365 underlying genes might be identified using a publicly available maize genome database (http://www.maizesequence.org), and 354 candidate genes were identified. Among the 365 SNPs, 185 SNPs were located within the coding region of the candidate genes, whereas the remaining 180 SNPs were located closer to the candidate genes, with a

Table 1 Joint linkage analysis of seven drought-related traits under the WW and WS conditions in the CN-NAM population

\begin{tabular}{|c|c|c|c|c|c|c|c|c|}
\hline \multirow[t]{2}{*}{ Trait } & \multicolumn{3}{|l|}{ WW } & \multicolumn{3}{|l|}{ WS } & \multicolumn{2}{|l|}{ WW-WS } \\
\hline & QTL number & PVE & $\mathrm{H}^{2}$ & QTL number & PVE & $\mathrm{H}^{2}$ & Shared QTL number & $P V E_{w w} / P V E_{W S}$ \\
\hline ASI & 15 & 48.1 & 61.7 & 11 & 42.8 & 57.3 & 4 & $26.2 / 22.9$ \\
\hline $\mathrm{PH}$ & 21 & 57.9 & 87.2 & 20 & 54.7 & 75.9 & 11 & $45.5 / 41.9$ \\
\hline GYPP & 8 & 23.7 & 68.9 & 8 & 20.2 & 49.3 & 0 & $0 / 0$ \\
\hline EL & 15 & 53.7 & 81.5 & 11 & 36.1 & 67.2 & 4 & $34.1 / 24.9$ \\
\hline HKW & 23 & 66.3 & 89.4 & 18 & 55.4 & 83.5 & 11 & $50.9 / 42.0$ \\
\hline KNPR & 13 & 34.7 & 77.7 & 10 & 28.1 & 61.3 & 1 & $7.7 / 6.1$ \\
\hline EW & 13 & 47.3 & 79.5 & 13 & 34.6 & 56.5 & 4 & $26.5 / 17.6$ \\
\hline Total & 108 & - & - & 91 & - & - & 35 & - \\
\hline
\end{tabular}

PVE phenotypic variation explained by all QTLs (\%), $H^{2}$ broad-sense heritability (\%), WW-WS common QTLs detected under WW and WS, $P V E_{W W}$ phenotypic variation explained by common QTLs under WW (\%), PVE $E_{W S}$ phenotypic variation explained by common QTLs under WS (\%) 

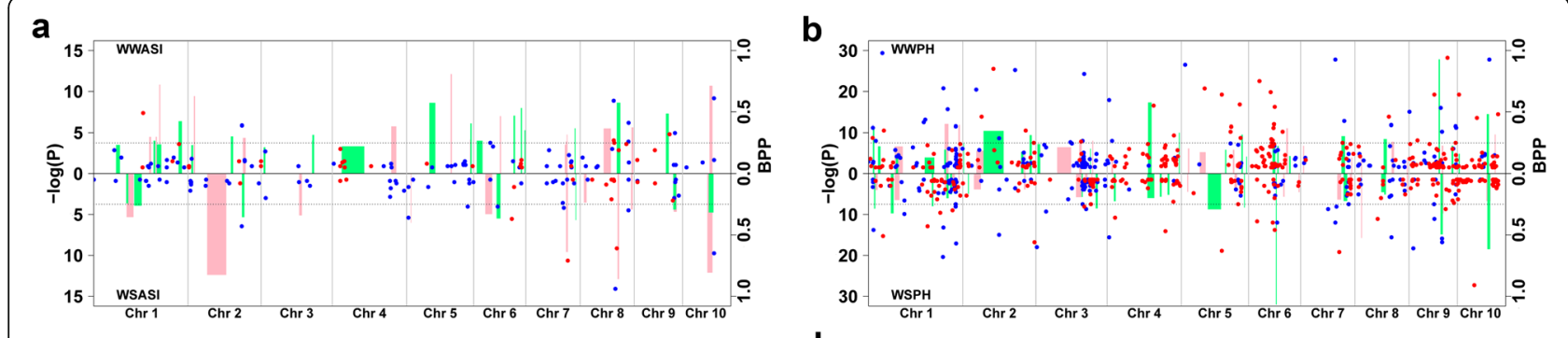

C

d
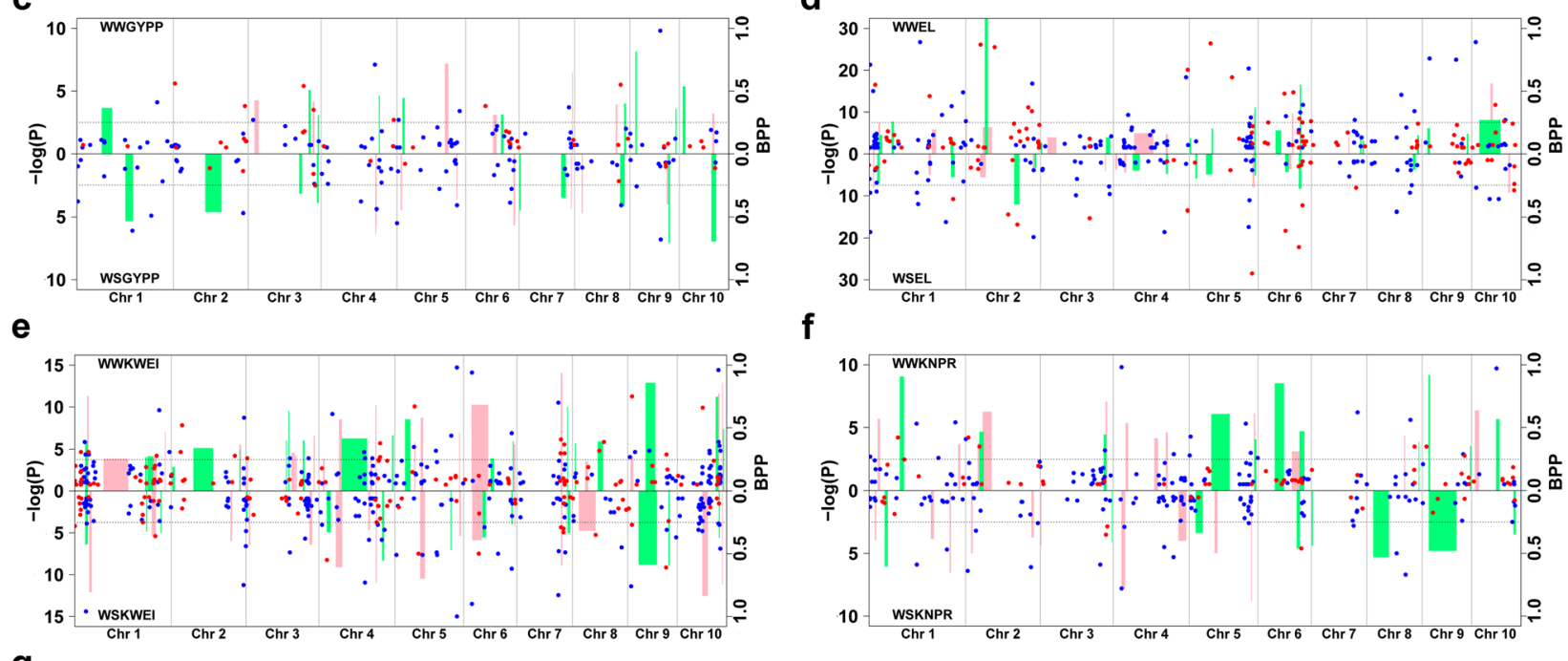

g

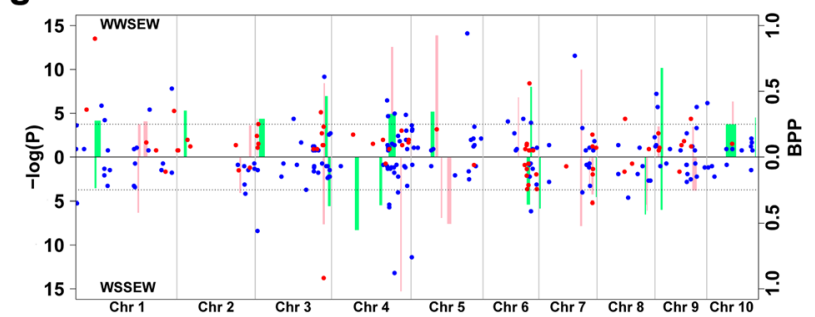

Fig. 1 Comparison between the results of the joint linkage analysis and GWAS for the seven drought-related traits under the WW and WS conditions for the CN-NAM and US-NAM populations. The pink and green bars represent the joint QTLs for CN-NAM and US-NAM, respectively. The bar width represents the support interval of the QTLS. Blue and red dots represent the significantly associated SNPs for CN-NAM and US-NAM, respectively. a ASI, b plant height $(\mathrm{PH})$, c grain yield per plant (GYPP), $\mathbf{d}$ ear length (EL), e hundred kernels weight (HKW), $\mathbf{f}$ kernel number per row (KNPR), and $\mathbf{g}$ ear weight (EW)

physical distance between the SNPs and the candidate genes ranging from 1 to 172,496 bp based on the B73 reference genome v2 (Additional file 10).

To validate the candidate genes associated with drought tolerance, we used the significantly differentially expressed genes obtained through the RNA-seq of the ovaries during flowering from the inbred line B73 under the WW and WS environments. Among the 354 candidate genes, 52 genes showed significantly different expression under the two water treatments, including 25 up-regulated genes and 27 down-regulated genes (Table 2).

\section{Genomic predictions of drought-related traits}

Figure 2a shows the accuracy of the genomic predictions for all of the target traits evaluated under the WW and
WS conditions using GBS data in the CN-NAM population. The prediction accuracy differed among all of the predicted traits for both water regimes. The accuracy values under WW ranged from 0.51 to 0.80 , with a mean of 0.64 , whereas the values under WS ranged from 0.40 to 0.74 , with a mean of 0.57. Except for the ASI, the accuracy values of other traits under WS were consistently lower than those under WW. Reflecting differences in the heritability and genetic architecture of the target traits, the GYPP and KNPR had small prediction accuracy under the same water regime compared with other agronomic traits. In addition, we conducted genomic prediction for all traits under the WW and WS conditions in the US-NAM population (Fig. 2b). Moreover, similar trends were observed between the two populations, although 
Table 2 List of SNPs significantly associated with seven drought-related traits and the closest candidate genes with significantly differential expression between the samples under the WW and WS conditions in B73

\begin{tabular}{|c|c|c|c|c|c|c|c|c|}
\hline Chr & $\begin{array}{l}\text { Position } \\
(\mathrm{bp})^{\mathrm{a}}\end{array}$ & BPP & $\begin{array}{l}\text { Inside } \\
\text { QTL }^{b}\end{array}$ & $\begin{array}{l}\text { Trait } \\
\text { affected }\end{array}$ & Gene ID & $\begin{array}{l}\text { Proximity of SNP } \\
\text { to gene (bp) }\end{array}$ & Gene function & $\mathrm{DEG}$ \\
\hline 1 & 4606329 & 0.28 & - & HKW & GRMZM2G341934 & 1721 & Peroxidase superfamily protein & 0.05 \\
\hline 1 & 27627382 & 0.27 & + & $\mathrm{PH}$ & GRMZM2G111324 & Inside gene & O-Glycosyl hydrolases family 17 protein & 0.07 \\
\hline 1 & 35465166 & 0.39 & + & HKW & GRMZM2G164562 & Inside gene & Chorismate synthase & 0.56 \\
\hline 1 & 154107233 & 0.53 & - & KNPR & GRMZM2G056039 & 25433 & Heat shock protein 70 & 2.01 \\
\hline 1 & 163956308 & 0.49 & - & ASI & GRMZM2G443525 & 130508 & ADR1-like 1 & 3.92 \\
\hline 1 & 219379659 & 0.25 & + & HKW & GRMZM2G146278 & 1444 & Cytochrome B561-1 & 0.24 \\
\hline 1 & 269328425 & 0.36 & - & EL & GRMZM2G157727 & Inside gene & Phytochrome A & 3.15 \\
\hline 1 & 275974364 & 0.30 & - & $\mathrm{PH}$ & GRMZM2G173852 & 590 & $\begin{array}{l}\text { Acyl-CoA N-acyltransferase with RING/FYVE/PHD-type } \\
\text { zinc finger protein }\end{array}$ & 1.52 \\
\hline 1 & 287727328 & 0.57 & - & $\mathrm{PH}$ & GRMZM2G133023 & 4891 & Stem-specific protein TSJT1 & 9.94 \\
\hline 2 & 10624855 & 0.26 & - & $\mathrm{EL}$ & GRMZM2G098239 & Inside gene & HXXXD-type acyl-transferase family protein & 0.05 \\
\hline 2 & 19262986 & 0.42 & - & KNPR & GRMZM2G162333 & 1756 & Pectin lyase-like superfamily protein & 0.02 \\
\hline 2 & 236797598 & 0.38 & - & GYPP & GRMZM2G016677 & 229 & Photosystem II subunit P-1/PsbP & 0.41 \\
\hline 3 & 17258723 & 0.56 & - & EW & GRMZM5G871336 & Inside gene & Expressed protein & 4.87 \\
\hline 3 & 165705047 & 0.51 & - & EL & GRMZM2G057823 & 17923 & $\begin{array}{l}\text { Aldolase superfamily protein/fructose-bisphospate aldolase } \\
\text { isozyme }\end{array}$ & 0.19 \\
\hline 3 & 197321346 & 0.38 & - & $\mathrm{HKW}$ & GRMZM2G021704 & Inside gene & Pyrimidin 4 & 0.33 \\
\hline 4 & 26425071 & 0.27 & - & $\mathrm{PH}$ & GRMZM2G089995 & Inside gene & Ethylene response factor $7 / \mathrm{AP} 2$ domain containing protein & 4.23 \\
\hline 4 & 172055239 & 0.62 & + & $\mathrm{EL}$ & GRMZM2G062084 & 260 & $\begin{array}{l}\text { P-loop containing nucleoside triphosphate hydrolases } \\
\text { superfamily protein/kinesin motor domain containing protein }\end{array}$ & 0.13 \\
\hline 4 & 204344848 & 0.38 & - & HKW & GRMZM2G117064 & 1105 & Long chain acyl-CoA synthetase 9 & 0.44 \\
\hline 4 & 218157821 & 0.31 & - & HKW & GRMZM2G422464 & 2207 & HhH-GPD base excision DNA repair family protein & 2.24 \\
\hline 5 & 10273708 & 0.55 & - & GYPP & GRMZM5G833140 & Inside gene & CHASE domain containing histidine kinase protein & 2.59 \\
\hline 5 & 11908448 & 0.28 & - & KNPR & GRMZM2G157147 & Inside gene & Phosphatidylinositol-4-phosphate 5-kinase 1 & 0.16 \\
\hline 5 & 16321609 & 0.36 & - & ASI & GRMZM2G134980 & Inside gene & Chaperone protein dnaJ & 0.69 \\
\hline 5 & 22556254 & 0.88 & + & $\mathrm{PH}$ & GRMZM5G869246 & Inside gene & Kinesin motor family protein & 0.32 \\
\hline 5 & 85252655 & 0.50 & + & HKW & GRMZM2G104632 & 14 & Glyceraldehyde-3-phosphate dehydrogenase of plastid 1 & 0.30 \\
\hline 5 & 140093287 & 0.32 & - & $\mathrm{HKW}$ & GRMZM2G060253 & Inside gene & HMG (high mobility group) box protein & 0.03 \\
\hline 5 & 143975579 & 0.51 & - & $\mathrm{HKW}$ & GRMZM2G158313 & 1348 & Basic-leucine zipper (bZIP) transcription factor family protein & 0.01 \\
\hline 5 & 208637679 & 0.95 & + & EL & GRMZM2G081214 & Inside gene & Phosphate-responsive 1 family protein & 6.03 \\
\hline 6 & 9710805 & 0.26 & - & KNPR & GRMZM2G055238 & 60720 & Ureide permease 5 & 5.40 \\
\hline 6 & 58454978 & 0.50 & - & HKW & GRMZM2G473788 & 4959 & Expressed protein & 1.87 \\
\hline 6 & 82185973 & 0.66 & + & $\mathrm{PH}$ & GRMZM2G430680 & Inside gene & Glucan synthase-like 12 & 0.34 \\
\hline 6 & 85797877 & 0.25 & + & $\mathrm{PH}$ & GRMZM2G365961 & 52 & Prephenate dehydrogenase family protein & 0.15 \\
\hline 6 & 88935877 & 0.40 & + & $\mathrm{PH}$ & GRMZM2G150302 & 13001 & $\begin{array}{l}\text { Nucleotide-diphospho-sugar transferases superfamily } \\
\text { protein/glycosyltransferase family } 43 \text { protein }\end{array}$ & 0.41 \\
\hline 6 & 119695193 & 0.50 & - & $\mathrm{HKW}$ & GRMZM2G368678 & Inside gene & Binding/expressed protein & 1.80 \\
\hline 6 & 121945548 & 0.49 & - & EL & GRMZM2G142409 & Inside gene & VIRB2-interacting protein 2/reticulon domain containing protein & 5.21 \\
\hline 6 & 150839908 & 0.41 & - & EL & GRMZM2G178797 & 1986 & Guanylyl cyclase 1 & 2.11 \\
\hline 6 & 155438561 & 0.28 & + & GYPP & GRMZM2G117344 & 12391 & Expressed protein & 0.18 \\
\hline 7 & 135573138 & 0.64 & - & $\mathrm{PH}$ & GRMZM2G130959 & 140 & Bug22p-like protein & 2.47 \\
\hline 7 & 152001464 & 0.27 & - & EL & GRMZM2G105750 & 36998 & ATP binding;protein kinases;protein serine/threonine kinases & 3.08 \\
\hline 7 & 157758647 & 0.33 & + & $\mathrm{HKW}$ & GRMZM2G129354 & Inside gene & ARF-GAP domain 5/GTPase-activating protein & 3.00 \\
\hline 7 & 160601468 & 0.37 & + & HKW & GRMZM2G058197 & Inside gene & $\mathrm{C} 2 \mathrm{H}$ 2-like zinc finger protein & 1.85 \\
\hline
\end{tabular}


Table 2 List of SNPS significantly associated with seven drought-related traits and the closest candidate genes with significantly differential expression between the samples under the WW and WS conditions in B73 (Continued)

\begin{tabular}{|c|c|c|c|c|c|c|c|c|}
\hline 8 & 129080393 & 0.61 & + & ASI & GRMZM2G171781 & 4780 & MYB domain protein 61 & 0.03 \\
\hline 8 & 148509016 & 0.56 & - & KNPR & GRMZM2G013625 & Inside gene & Associated molecule with the SH3 domain of STAM 2 & 3.20 \\
\hline 8 & 166653395 & 0.26 & + & ASI & GRMZM2G143640 & Inside gene & MYB family transcription factor & 20.26 \\
\hline 8 & 166781122 & 0.30 & + & ASI & GRMZM5G805609 & Inside gene & Glycosyl hydrolase superfamily protein & 4.33 \\
\hline 9 & 24062413 & 0.35 & + & KNPR & GRMZM2G082855 & 565 & ERECTA-like 2/receptor-like protein kinase 5 precursor & 0.34 \\
\hline 9 & 24062931 & 0.61 & - & $\mathrm{PH}$ & GRMZM2G082855 & 47 & ERECTA-like 2/receptor-like protein kinase 5 precursor & 0.34 \\
\hline 9 & 31283082 & 0.26 & - & GYPP & GRMZM2G308595 & 76352 & Nudix hydrolase homolog 21 & 8.39 \\
\hline 9 & 78421440 & 0.32 & + & HKW & GRMZM2G094497 & Inside gene & ATPase, V1 complex, subunit B protein & 0.58 \\
\hline 9 & 133586941 & 0.31 & - & HKW & GRMZM2G317262 & 252 & F-box family protein & 6.68 \\
\hline 9 & 134721582 & 0.94 & - & $\mathrm{PH}$ & GRMZM2G033846 & 250 & $\mathrm{Ca} 2+$-binding protein $1 /$ EF hand family protein & 0.05 \\
\hline 10 & 6398185 & 0.37 & + & $\mathrm{PH}$ & GRMZM2G057753 & Inside gene & Ovate family protein 13/DUF623 domain containing protein & 0.10 \\
\hline 10 & 15549436 & 0.64 & - & $\mathrm{PH}$ & GRMZM2G088689 & Inside gene & $\begin{array}{l}\text { Thiamin diphosphate-binding fold (THDP-binding) superfamily } \\
\text { protein/dehydrogenase E1 component domain containing } \\
\text { protein }\end{array}$ & 5.47 \\
\hline
\end{tabular}

aphysical position based on B73 maize reference genome v2

b " +" repesents that significantly assiciated SNP is located in QTL support interval; "-" repesents that significantly assiciated SNP is not located in QTL support interval 'differential expressed genes (DEG),expression level ratios of candidate genes between WS and WW from B73

lower accuracy was observed for the US-NAM population compared with the CN-NAM population.

\section{Discussion}

In the present study, we assessed the phenotypes of seven important agronomic traits associated with drought stress for two sets of NAM populations under well-watered and water-stressed environments. Although the CN-NAM population, which included 1972 lines, was evaluated for only 2 years at a single location, the broad-sense heritability estimates based on plots for each trait across the 11 CN-NAM families were moderately high (Table 1 ). These estimates of heritability suggested that variations for all of the target traits in the CN-NAM population were primarily influenced through QTLs. The US-NAM population, which included 2948 lines, was phenotyped for only 1 year under the different environments. The US-NAM population represented a validation population and was used to verify the results obtained in the CN-NAM population.
The linkage analysis within a single bi-parental population was successfully used to locate QTLs that affect drought tolerance in maize. In the present study, both NAM populations with much larger mapping population sizes and high-density genetic maps were used to perform the first detection of QTLs that control drought tolerance. A total of 169 joint QTLs were detected under the water-stressed environment in the both NAM populations. These drought-related QTLs were compared with previously reported meta-QTLs (mQTLs) (Additional file 11), which provided a good summary of the published QTLs associated with the drought tolerancerelated traits involved in the present study. Among 169 drought-related QTLs, 106 QTLs overlapped with at least one mQTL and six QTLs overlapped with three mQTLs obtained in three different studies. One genomic region on chromosome 1 (43.75-46.55 Mb) harbored one QTL for WSHKW in the CN-NAM population and one QTL for WSKNPR in the US-NAM population, and it also
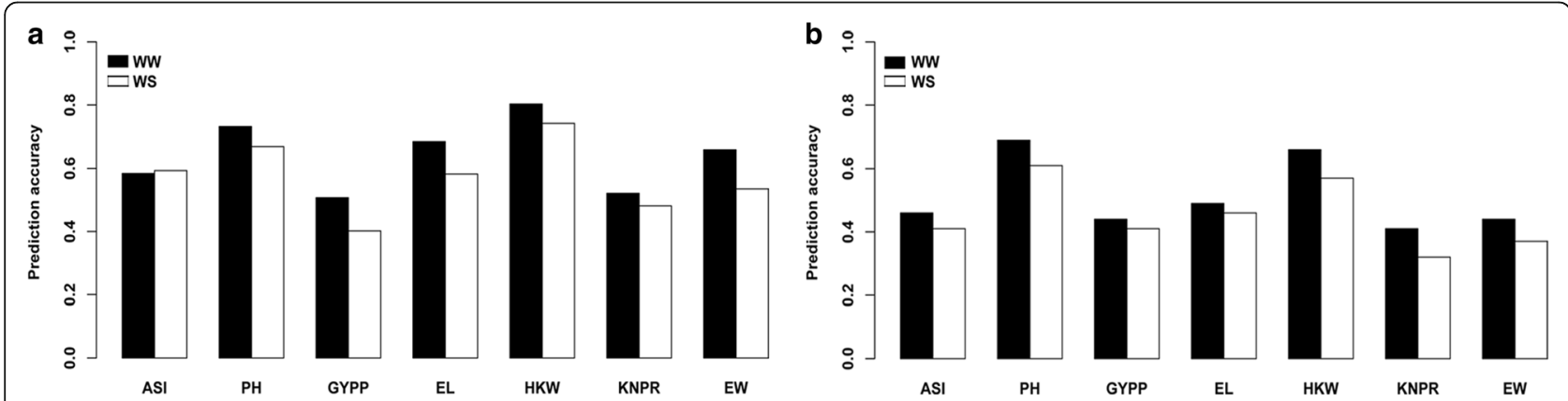

Fig. 2 Prediction accuracy of seven drought-related traits under WW and WS conditions using genome-wide SNPs in the CN-NAM (a) and US-NAM (b) populations 
contained three mQTLs that were also reported in the studies of Li et al. [14], Semagn et al. [15] and Almeida et al. [12]. Particularly, Li et al. [14] identified the candidate gene $p d c 3$ as associated with drought tolerance in this region through a bioinformatics analysis. The region between 204.65 and $206.18 \mathrm{Mb}$ on chromosome 3 included three QTLs for WSHKW, WSEL and WSEW in the CN-NAM population. In this physical region, Almeida et al. [12] detected an mQTL that affects the number of ears per plant under WS conditions, suggesting that this region is an important drought tolerance locus that controls grain yield. Another important genomic region located in the 85.3295.89 $\mathrm{Mb}$ interval on chromosome ten overlapped with three QTLs for WSHKW and WSASI in the CN-NAM population and WSASI in the US-NAM population. Almeida et al. [11] and Li et al. [14] reported two mQTLs in this region. In addition, the gene $Z m S N A C 1$, which is responsive to drought stress in maize [27], was located in this region. The genomic regions described above provide important target regions for identifying candidate genes associated with drought stress and marker-assisted introgression for drought tolerance in maize.

The fifty-two candidate genes identified in the present study were identified as encoding transcription factors, signal transduction factors, dehydrins and osmotins, etc. (Table 2). These genes have also been frequently associated with drought tolerance in plants. For example, the GRMZM2G081214 gene was associated with the most highly robust SNP $(\mathrm{BPP}=0.95)$ located in the QTL region. This gene is predicted role in ABA activity and encodes a phosphate-responsive 1 family protein (http:// www.maizegdb.org/). The gene is more differentially upregulated in the ovaries of the inbred line B73 under WS compared with WW.

Two robust associated SNPs were located near the GRMZM2G082855 gene, which is associated with plant height and kernel number per row. This gene encodes the precursor to receptor-like protein kinase 5, a plant protein kinase responsive to abiotic stress. Receptor-like kinase (RLK) is widespread in plants, particularly in Arabidopsis and rice, and includes 600 and 1132 RLKs, respectively [28].

Two genes, GRMZM2G143640 and GRMZM5G805609, were associated with the ASI and harbored one strong associated SNP in the ASI QTL regions on chromosome 8, respectively. GRMZM2G143640 encodes a DIVARICATAlike putative MYB DNA-binding domain superfamily transcript factor that plays an important role in plant growth and development and responds to abiotic stress, including drought tolerance [29]. This candidate gene was more differentially up-regulated under WS than WW in the inbred line B73. GRMZM5G805609 encodes glucan endo-1,3-beta-glucosidase 7 of the glycosyl hydrolase protein superfamily, and it was also significantly up-regulated in the inbred line B73 under drought stress.

Most of the remaining genes listed in Table 2 were associated with grain yield component traits, which are highly associated with drought tolerance. A full understanding of the genetic control of these traits would be helpful for maize breeding for high yields under watered-well or water-stressed environments.

With the advancements of next-generation sequencing and statistical models, maize breeders have successfully used genomic prediction methods to estimate the breeding value of unphenotyped lines within breeding populations [30], single bi-parental or multiple bi-parental populations [31, 32] and association populations [33]. Genomic prediction models have been demonstrated as advantageous for complex traits controlled through many small-effects loci, such as grain yield [34, 35]. In the present study, good prediction accuracies were obtained for the target traits under WS, although certain traits showed low heritability. We observed that trait heritability affects the prediction accuracy of the $\mathrm{CN}$ NAM population. The target traits with high heritability generally achieved high prediction accuracy under the WS or WW conditions. Marker density has previously been demonstrated as an important factor for prediction accuracy [36, 37]. We selected 100, 300, 500, 1000, 2000, 5000,10000 or 20000 SNPs that evenly covered the entire genome to investigate the influence of marker density on the prediction accuracy of the CN-NAM population (Fig. 3). The results indicated that approximately 5000 SNP markers were sufficient to achieve accurate predictions for the drought-related traits under the WW or WS environments. Zhang et al. [38] used low-density SNPs and high-density markers (GBS) to predict the grain yield, flowering time and plant height for 19 bi-parental populations under WW and WS treatments, and their results indicate that moderate marker density was largely sufficient for complex and simple traits. Genomic predictions for drought-related traits are efficient in multiple bi-parental populations, such as the CN-NAM population. However, additional studies are needed to assess the prediction accuracy for drought tolerance within association populations with unrelated lines.

\section{Conclusions}

In this study, a total of 220 QTLs under well-watered conditions and 169 QTLs under water-stressed conditions were detected by joint linkage mapping in the $\mathrm{CN}$ NAM and US-NAM populations. The genome-wide association analysis identified 365 SNPs associated with drought-related traits, and these SNPs were located in 354 candidate genes. Of these candidate genes, 52 candidate genes showed significant differential expression in the inbred line B73 under the well-watered and water- 


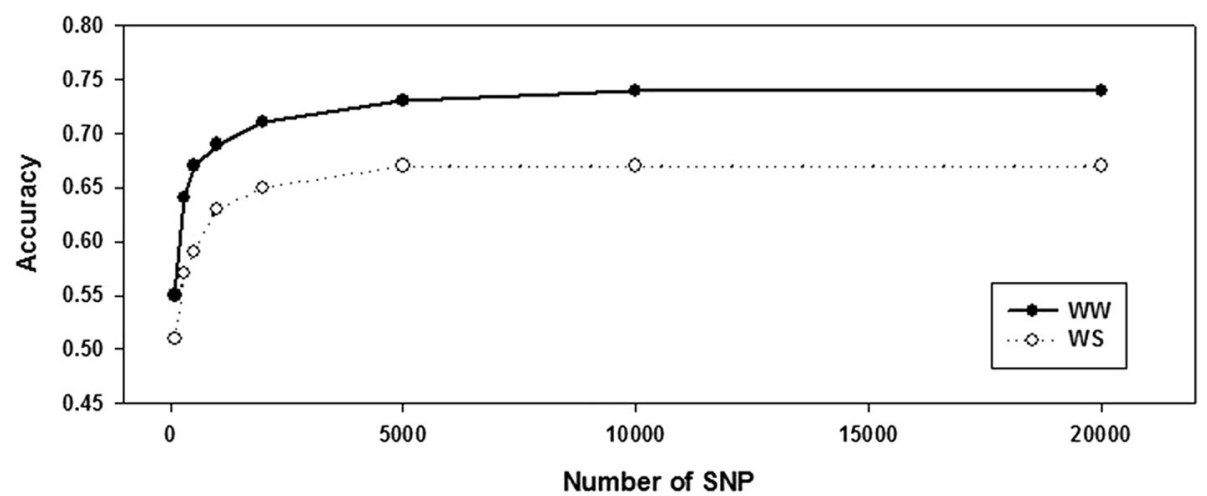

Fig. 3 Accuracy of the whole-genome predictions of PH under the WW and WS conditions within the CN-NAM population depending on the number of SNPs. Accuracies averaged over 20 cross-validation runs are shown for 100, 300, 500, 1000, 2000, 5000, 10000 and 20000 evenly spaced SNPS

stressed conditions. In addition, the moderate-density SNPs obtained through genotyping-by-sequencing were able to make accurate predictions in the nested association mapping population for drought-related traits with moderate-to-high heritability under the water-stressed conditions. This paper presents these drought tolerance QTLs and candidate genes for the maize scientific community to provide detailed direction for future studies.

\section{Methods}

\section{Plant materials and field environments}

The CN-NAM and US-NAM panels were generated as previously described [20]. A total of 1972 CN-NAM lines were grown and measured in the spring of 2009 and 2010 in Urumqi of Xinjiang Province, where the institute of crop science belonging to the Chinese Academy of Agricultural Sciences has set up experimental field bases. The institute of crop science was approved for field experiments, and the field studies did not involve endangered or protected species. For each year, all of the plant materials were subjected to well-watered (WW) and water-stressed (WS) treatments. Trials of each treatment were planted in single-row plots of 11 plants, and there were two replications. Two irrigation regimes were applied using the furrow irrigation method starting at the seeding period. In the WW regime, irrigation was provided in 15-day intervals. In the WS regime, irrigation was given until 3 weeks prior to the expected anthesis date in each CN-NAM family. This stress condition was maintained until 4 weeks after $50 \%$ of the RILs flowered. Nineteen of the 25 US-NAM families, including 2948 RILs, were grown in Urumqi, Xinjiang Province in the spring of 2013. Based on the previous flowering time data [20], four families (CML228, CML247, CML52, and IL14H) with late maturity were excluded from the drought tolerance identification. The P39 and Hp301 families belonging to the sweet and popcorn types were also excluded in the present study. Single-row plots of 11 plants with one replication were grown for each RIL under the WW and WS environments. Each plot was $3 \mathrm{~m}$ in length, and the rows were spaced $0.6 \mathrm{~m}$ apart. The RIL families were randomly arranged as previously described [39]. Within each family, incomplete blocks consisting of 40 random RILs, the B73 line and alternate parents of the family were planted in an alpha lattice. In the WS regime, the drip irrigation method was applied in 10-day intervals until 3 and 5 weeks prior to the expected anthesis date and after $50 \%$ anthesis occurred. For the WW condition, the soil moisture was maintained at field capacity. All plant materials used in this study were conserved in our experiment lab and we declared that all plant materials used in this study comply with the "Convention on the Trade in Endangered Species of Wild Fauna and Flora".

\section{Phenotyping}

A total of seven traits were measured for all of the lines under both water regimes. The anthesis-silking interval (ASI) was counted as the difference (in days) between male and female flowering times in each plot. The plant height $(\mathrm{PH})$ was calculated as the average height of five random plants measured from the ground to the tassel tip in each plot. Five representative plants in each plot were harvested. The grain yield per plant (GYPP) was evaluated from an average of five plants. The ear length (EL), kernel number per row (KNPR), and ear weight (EW) were measured for five ears and averaged over the plot. The hundred kernel weight (HKW) was estimated from the average weight of 100 randomly selected seeds in three samples.

\section{Genotypic data}

A set of 0.95 million SNPs from the CN-NAM and USNAM populations was generated using Genotyping-By- 
Sequencing technology [40]. The missing SNPs were imputed using the FILLIN method in TASSEL v.5.0 [41]. These marker sets are publicly available at http:// www.panzea.org. The SNP sites exhibiting more than $20 \%$ missing taxa, $<5 \%$ minor allelic frequency (MAF), and $>20 \%$ heterozygosity were excluded from the raw genotype datasets. Thus, a total of 333,577 and 404,543 SNPs were identified for the CN-NAM and US-NAM populations, respectively. These SNPs were subsequently used in the GWAS and genomic predictions. For the joint linkage mapping, two composite genetic maps that included 4932 and 5296 markers were constructed for the CN-NAM and US-NAM populations, respectively. Detailed information on the maps and genotypes scores has previously been described [20].

\section{Phenotypic data analysis}

For the CN-NAM and US-NAM populations, the best linear unbiased prediction (BLUP) for all of the traits of each line across environments (CN-NAM) and within a single environment (US-NAM) was calculated from a random effects model using PROC MIXED in SAS 9.2. In models across the environments of the CN-NAM population, the environment, family, family*environment and entry (family) were considered random effects. The mixed model was fitted within a single environment of the US-NAM population, and the family, RIL within family, blocks, rows and columns were included in the field design. Correlation coefficients were obtained based on the BLUP using Pearson's statistic applied using the cor function of $\mathrm{R}$ software. The broad-sense heritability $\left(h^{2}\right)$ for each trait across environments in the CN-NAM population was calculated on a plot basis using the ANOVA tool in QTL IciMapping Version 3.3 [42].

\section{Joint linkage mapping in CN-NAM and US-NAM}

The joint linkage analysis for CN-NAM and US-NAM was conducted in SAS 9.2. The detailed information for joint linkage mapping has previously been described [21]. Briefly, PROC GLMSelect was implemented to select the significant marker effects in a family-nested QTL model. For all of the traits, the $P$-values for the entry and exit of the model were determined using permutation testing. The phenotypic variation explained by all of the QTLs was counted according to Li [43]. The joint linkage QTL support intervals were counted according to Tian [22].

\section{GWAS in CN-NAM and US-NAM}

The GWAS was performed in a single NAM population using the Fixed and random model Circulating Probability Unification (FarmCPU) method in $\mathrm{R}$ software [44]. To identify the SNPs with the most robust associations with phenotypes, a subsampling procedure was used in the GWA analysis [45]. For each subsampling, $80 \%$ of the RILs of each NAM population were randomly sampled without replacement. This procedure was repeated 100 times between each trait and polymorphism. In each subsampling, we implemented a Bonferroni-corrected threshold probability of $0.05 / \mathrm{N}$ to verify the significance levels, where $\mathrm{N}$ is the number of individual trait-SNP combinations tested. The significance levels were used to control the false positives in the GWAS. The bootstrap posterior probability (BPP), which is defined as the proportion of times that SNPs were included in the 100 subsamples, was calculated for each significantly associated SNP. Only SNPs with BPP $\geq 0.05$ were listed in the results. According to Valdar et al. [45], a BPP $\geq$ 0.25 was considered the most robust SNP association.

\section{Validation of the candidate genes using RNA-seq data}

Based on the maize B73 reference genome assembly V2, genes co-localizing with or adjacent to the associated SNPs were determined to be candidate genes for drought tolerance. Functional annotations of the candidate genes were conducted using blastp, conserved domain search tools, the Maize Genome Database and a literaturespecific inspection for each gene. To validate the candidate genes for drought tolerance revealed in the GWAS, the available RNA-seq data of the inbred line B73 was used to evaluate the expression of candidate genes under the WW and WS environments [46]. The RNA-seq data from pollinated ovaries of drought sensitive inbred B73 under WWW and WS environments were downloaded from the NCBI web site (http://www.ncbi.nlm.nih.gov/sra/). Briefly, after obtaining the RNA-seq data, the quality control of the raw reads were dealt with the FASTX toolkit (http://hannonlab.cshl.edu/fastx_toolkit/). High-quality RNA-seq reads were mapped to the maize B73 reference genome (B73 AGPv2; http://www.maizesequence.org) using the programs TopHat v2.0.4 [47]. Differential expression analysis was conducted using the HTSeq-DEseq workflow [48]. A false discovery rate (FDR) $<0.05$ after BenjaminiHochberg correction for multiple tests was applied.

\section{Cross-validated genomic prediction}

We conducted genomic predictions using a mixedmodel solver in the rrBLUP package [49] distributed by $\mathrm{R}$ software. The prediction accuracy of all traits was evaluated through cross-validation. To perform the cross-validation, a five-fold cross-validation scheme was applied and repeated 20 times. All of the lines in the NAM panel were randomly divided into five disjointed subsets. One of five subsets was selected as the validation population, and the remaining four subsets were used as the training population to estimate the SNP effects for predicting the lines' values in the validation. The prediction accuracy was calculated according to Pearson's 
correlation between the predicted and observed values averaged over all of the cross-validations and replicates.

\section{Additional files}

Additional file 1: Table S1. Statistical analysis of the seven droughtrelated traits' BLUP values under the WW and WS conditions within the CN-NAM population. (DOCX $19 \mathrm{~kb}$ )

Additional file 2: Table S2. Statistical analysis of the seven droughtrelated traits value under WW and WS within the US-NAM population. (DOCX 19 kb)

Additional file 3: Table S3. Correlation of the seven drought-related traits under the WW (above diagonal) and WS (under diagonal) conditions within the CN-NAM population. (DOCX $19 \mathrm{~kb}$ )

Additional file 4: Table S4. Correlation of the seven drought-related traits under the WW (above diagonal) and WS (under diagonal) conditions within the US-NAM population. (DOCX $19 \mathrm{~kb}$ )

Additional file 5: Joint linkage analysis summary for the seven droughtrelated traits under the WW and WS conditions in the CN-NAM population. (XLSX 65 kb)

Additional file 6: Joint linkage analysis summary for the seven droughtrelated traits under the WW and WS conditions in the US-NAM population. (XLSX 79 kb)

Additional file 7: Table S5. Joint QTL validation between the CN-NAM and US-NAM populations under the WW and WS conditions. (DOCX $18 \mathrm{~kb}$ )

Additional file 8: Significantly associated SNPS at the threshold of $\mathrm{BPP}=0.05$ for all of the traits under the WW and WS conditions in the CN-NAM population. (XLSX $126 \mathrm{~kb}$ )

Additional file 9: Significantly associated SNPS at the threshold of $\mathrm{BPP}=0.05$ for all of the traits under the WW and WS conditions in the US-NAM population. (XLSX $83 \mathrm{~kb}$ )

Additional file 10: All of the robust SNPS associated with the seven drought-related traits and the closest candidate genes in the two NAM populations. (XLSX $40 \mathrm{~kb})$

Additional file 11: Comparison between the QTL results identified for the seven drought-related traits under WS in both NAM populations and the reported $\mathrm{mQTLS}$ associated with drought tolerance. (XLSX $19 \mathrm{~kb}$ )

\section{Abbreviations}

ASI: Anthesis-silking interval; BLUP: Best linear unbiased prediction; BPP: Bootstrap posterior probability; CN-NAM: Chinese nested association mapping; EL: Ear length; EW: Ear weight; GWAS: Genome-wide association studies; GYPP: Grain yield per plant; HKW: Hundred kernel weight; KNPR: Kernel number per row; PH: Plant height; US-NAM: United State nested association mapping; WS: Water-stressed; WW: Well-watered

\section{Funding}

This work was partly supported by National Natural Science Foundation (U1138304),the Agricultural Ministry of China (2014ZX08003004, 2015NWB030-04), the Ministry of Science and Technology of China (2011DFA30450, 2013BAD01B02), the CAAS Innovation Program, an Emerging Research Issues Internal Competitive Grant from the Agricultural Research Center at Washington State University, College of Agricultural, Human, and Natural Resource Sciences, the Endowment and Research Project (No. 126593) from the Washington Grain Commission.

\section{Availability of data and materials}

All publicly available genotypic data supporting the results of this article are available in the Panzea Date Sets (accession number: ZeaGBSv2.7) at http:// www.panzea.org. The original RNA-seq dataset of the inbred B73 under well-watered and water-stressed conditions are available in NCBI Sequence Read Archive (SRA) project SRP014792 (http://www.ncbi.nlm.nih.gov/sra/).

\section{Authors' contributions}

$\mathrm{CL}$ analyzed the data and drafted the manuscript. YL participated in the study design and analyzed the data. SB and LC provided phenotypic information.
$X W, Z D, S Y$ and SY participated in the data collection. ESB, ZZ, TW, and YL conceived of the study, managed the project design and coordination, collected data, and helped to draft the manuscript. All authors read and approved the final manuscript.

\section{Competing interests}

The authors declare that they have no competing interests.

\section{Consent for publication}

Not applicable.

Ethics approval and consent to participate

Not applicable.

\section{Author details}

${ }^{1}$ Institute of Crop Science, Chinese Academy of Agricultural Sciences, Beijing 100081, China. ${ }^{2}$ Institute of Food Crops, Xinjiang Academy of Agricultural Sciences, Urumqi 830000, China. ${ }^{3}$ Institute for Genomic Diversity, Cornell University, Ithaca, NY, USA. ${ }^{4}$ USA Department of Agriculture-Agricultural Research Service, Ithaca, NY, USA. ${ }^{5}$ Department of Crop and Soil Sciences, Washington State University, Pullman, WA, USA.

Received: 7 May 2016 Accepted: 18 October 2016

Published online: 08 November 2016

\section{References}

1. Tuberosa R, Salvi S. Genomics-based approaches to improve drought tolerance of crops. Trends Plant Sci. 2006;11(8):405-12.

2. Bruce WB, Edmeades GO, Barker TC. Molecular and physiological approaches to maize improvement for drought tolerance. J Exp Bot. 2002;53(366):13-25.

3. Bolanos J, Edmeades GO. The importance of the anthesis-silking interval in breeding for drought tolerance in tropical maize. Field Crop Res. 1996;48(1): 65-80.

4. Frova C, Krajewski P, di Fonzo N, Villa M, Sari-Gorla M. Genetic analysis of drought tolerance in maize by molecular markers I. Yield components. Theor Appl Genet. 1999;99(1-2):280-8.

5. Setter TL. Analysis of constituents for phenotyping drought tolerance in crop improvement. Front Physiol. 2012;3:180.

6. Tuberosa R. Phenotyping for drought tolerance of crops in the genomics era. Front Physiol. 2012;3:347.

7. Ziyomo C, Bernardo R. Drought tolerance in maize: indirect selection through secondary traits versus genome wide selection. Crop Sci. 2013; 53(4):1269-75.

8. Messina CD, Podlich D, Dong ZS, Samples M, Cooper M. Yield-trait performance landscapes: from theory to application in breeding maize for drought tolerance. J Exp Bot. 2011;62(3):855-68.

9. Ribaut JM, Hoisington DA, Deutsch JA, Jiang C, GonzalezdeLeon D. Identification of quantitative trait loci under drought conditions in tropical maize. II. Flowering parameters and the anthesis-silking interval. Theor Appl Genet. 1996;92(7):905-14.

10. Sari-Gorla M, Krajewski P, Di Fonzo N, Villa M, Frova C. Genetic analysis of drought tolerance in maize by molecular markers. II. Plant height and flowering. Theor Appl Genet. 1999;99(1-2):289-95.

11. Almeida GD, Makumbi D, Magorokosho C, Nair S, Borem A, Ribaut JM, Banziger M, Prasanna BM, Crossa J, Babu R. QTL mapping in three tropical maize populations reveals a set of constitutive and adaptive genomic regions for drought tolerance. Theor Appl Genet. 2013;126(3):583-600.

12. Almeida GD, Nair S, Borem A, Cairns J, Trachsel S, Ribaut JM, Banziger M, Prasanna BM, Crossa J, Babu R. Molecular mapping across three populations reveals a QTL hotspot region on chromosome 3 for secondary traits associated with drought tolerance in tropical maize. Mol Breed. 2014;34(2):701-15.

13. Hao ZF, Li XH, Liu XL, Xie CX, Li MS, Zhang DG, Zhang SH. Meta-analysis of constitutive and adaptive QTL for drought tolerance in maize. Euphytica. 2010:174(2):165-77.

14. Li W, Liu Z, Shi Y, Song Y, Wang T, Xu C, Li Y. Detection of consensus genomic region of QTLs relevant to drought tolerance in maize by QTL Meta-analysis and bioinformatics approach. Acta Agron Sin. 2010;36:1457-67.

15. Semagn K, Beyene Y, Warburton ML, Tarekegne A, Mugo S, Meisel B, Sehabiague P, Prasanna BM. Meta-analyses of QTL for grain yield and anthesis silking interval in 18 maize populations evaluated under waterstressed and well-watered environments. BMC Genomics. 2013;14. 
16. Lu YL, Zhang SH, Shah T, Xie CX, Hao ZF, Li XH, Farkhari M, Ribaut JM, Cao MJ, Rong TZ, et al. Joint linkage-linkage disequilibrium mapping is a powerful approach to detecting quantitative trait loci underlying drought tolerance in maize. Proc Natl Acad Sci U S A. 2010;107(45):19585-90.

17. Xue YD, Warburton ML, Sawkins M, Zhang XH, Setter T, Xu YB, Grudloyma P, Gethi J, Ribaut JM, Li WC, et al. Genome-wide association analysis for nine agronomic traits in maize under well-watered and water-stressed conditions. Theor Appl Genet. 2013;126(10):2587-96.

18. Thirunavukkarasu N, Hossain F, Arora K, Sharma R, Shiriga K, Mittal S, Mohan S, Namratha PM, Dogga S, Rani TS, et al. Functional mechanisms of drought tolerance in subtropical maize (Zea mays L.) identified using genome-wide association mapping. BMC Genomics. 2014;15:1182.

19. McMullen MD, Kresovich S, Villeda HS, Bradbury P, Li H, Sun Q, Flint-Garcia S, Thornsberry J, Acharya C, Bottoms C, et al. Genetic properties of the maize nested association mapping population. Science. 2009;325(5941): 737-40.

20. Li C, Li Y, Bradbury PJ, Wu X, Shi Y, Song Y, Zhang D, Rodgers-Melnick E, Buckler ES, Zhang $Z$, et al. Construction of high-quality recombination maps with low-coverage genomic sequencing for joint linkage analysis in maize. BMC Biol. 2015;13:78.

21. Buckler ES, Holland JB, Bradbury PJ, Acharya CB, Brown PJ, Browne C, Ersoz E, Flint-Garcia S, Garcia A, Glaubitz JC, et al. The genetic architecture of maize flowering time. Science. 2009;325(5941):714-8.

22. Tian F, Bradbury PJ, Brown PJ, Hung H, Sun Q, Flint-Garcia S, Rocheford TR, McMullen MD, Holland JB, Buckler ES. Genome-wide association study of leaf architecture in the maize nested association mapping population. Nat Genet. 2011;43(2):159-62.

23. Brown PJ, Upadyayula N, Mahone GS, Tian F, Bradbury PJ, Myles S, Holland JB, Flint-Garcia S, McMullen MD, Buckler ES, et al. Distinct genetic architectures for male and female inflorescence traits of maize. PLoS Genet. 2011;7(11), e1002383.

24. Cook JP, McMullen MD, Holland JB, Tian F, Bradbury P, Ross-lbarra J, Buckler ES, Flint-Garcia SA. Genetic architecture of maize kernel composition in the nested association mapping and inbred association panels. Plant Physiol. 2012;158(2): 824-34.

25. Peiffer JA, Romay MC, Gore MA, Flint-Garcia SA, Zhang Z, Millard MJ, Gardner CA, McMullen MD, Holland JB, Bradbury PJ, et al. The genetic architecture of maize height. Genetics. 2014;196(4):1337-56.

26. Peiffer JA, Flint-Garcia SA, De Leon N, McMullen MD, Kaeppler SM, Buckler ES. The genetic architecture of maize stalk strength. PLoS One. 2013;8(6), e67066.

27. Vilela B, Moreno-Cortes A, Rabissi A, Leung J, Pages M, Lumbreras V. The maize OST1 kinase homolog phosphorylates and regulates the maize SNAC1-type transcription factor. PLoS One. 2013;8(2), e58105.

28. Shiu SH, Karlowski WM, Pan R, Tzeng YH, Mayer KF, Li WH. Comparative analysis of the receptor-like kinase family in Arabidopsis and rice. Plant Cell. 2004;16(5):1220-34.

29. Osakabe Y, Osakabe K, Shinozaki K, Tran LS. Response of plants to water stress. Front Plant Sci. 2014;5:86

30. Crossa J, de Los Campos G, Perez P, Gianola D, Burgueno J, Araus JL, Makumbi D, Singh RP, Dreisigacker S, Yan JB, et al. Prediction of genetic values of quantitative traits in plant breeding using pedigree and molecular markers. Genetics. 2010;186(2):713-U406.

31. Guo Z, Tucker DM, Lu J, Kishore V, Gay G. Evaluation of genome-wide selection efficiency in maize nested association mapping populations. Theor Appl Genet. 2012;124(2):261-75.

32. Riedelsheimer C, Endelman JB, Stange M, Sorrells ME, Jannink JL, Melchinger AE. Genomic predictability of interconnected biparental maize populations. Genetics. 2013;194(2):493-503.

33. Rincent $R$, Laloe $D$, Nicolas $S$, Altmann T, Brunel D, Revilla P, Rodriguez VM, Moreno-Gonzalez J, Melchinger A, Bauer E, et al. Maximizing the reliability of genomic selection by optimizing the calibration set of reference individuals: comparison of methods in two diverse groups of maize inbreds (Zea mays L.). Genetics. 2012;192(2):715-28.

34. Jannink JL, Lorenz AJ, Iwata H. Genomic selection in plant breeding: from theory to practice. Brief Funct Genomics. 2010;9(2):166-77.

35. Burgueno J, de Los Campos G, Weigel K, Crossa J. Genomic prediction of breeding values when modeling genotype $\mathrm{x}$ environment interaction using pedigree and dense molecular markers. Crop Sci. 2012;52(2):707-19.

36. Poland JA, Brown PJ, Sorrells ME, Jannink JL. Development of high-density genetic maps for barley and wheat using a novel two-enzyme genotypingby-sequencing approach. PLoS One. 2012;7(2):e32253.
37. Crossa J, Beyene Y, Kassa S, Perez P, Hickey JM, Chen C, de Los Campos G, Burgueno J, Windhausen VS, Buckler E, et al. Genomic prediction in maize breeding populations with genotyping-by-sequencing. G3-Genes Genom Genet. 2013:3(11):1903-26.

38. Zhang X, Perez-Rodriguez P, Semagn K, Beyene Y, Babu R, Lopez-Cruz MA Vicente FS, Olsen M, Buckler $\mathrm{E}$, Jannink JL, et al. Genomic prediction in biparental tropical maize populations in water-stressed and well-watered environments using low-density and GBS SNPs. Heredity. 2015;114(3):291-9.

39. Hung HY, Browne C, Guill K, Coles N, Eller M, Garcia A, Lepak N, MeliaHancock S, Oropeza-Rosas M, Salvo S, et al. The relationship between parental genetic or phenotypic divergence and progeny variation in the maize nested association mapping population. Heredity. 2012;108(5):490-9.

40. Elshire RJ, Glaubitz JC, Sun Q, Poland JA, Kawamoto K, Buckler ES, Mitchell SE. A robust, simple genotyping-by-sequencing (GBS) approach for high diversity species. PLoS One. 2011;6(5), e19379.

41. Bradbury PJ, Zhang Z, Kroon DE, Casstevens TM, Ramdoss Y, Buckler ES. TASSEL: software for association mapping of complex traits in diverse samples. Bioinformatics. 2007;23(19):2633-5.

42. Wang J, Li H, Zhang L, Li C, Meng L. QTL iciMapping Software. Beijing: Isbreeding; 2012. Available: http://www.isbreeding.net

43. Li H, Bradbury P, Ersoz E, Buckler ES, Wang J. Joint QTL linkage mapping for multiple-cross mating design sharing one common parent. PLoS One. 2011;6(3), e17573

44. Liu X, Huang M, Fan B, Buckler ES, Zhang Z. Iterative usage of fixed and random effect models for powerful and efficient genome-wide association studies. PLoS Genet. 2016:12(2), e1005767.

45. Valdar W, Holmes CC, Mott R, Flint J. Mapping in structured populations by resample model averaging. Genetics. 2009;182(4):1263-77.

46. Kakumanu A, Ambavaram MM, Klumas C, Krishnan A, Batlang U, Myers E, Grene R, Pereira A. Effects of drought on gene expression in maize reproductive and leaf meristem tissue revealed by RNA-Seq. Plant Physiol. 2012;160(2):846-67.

47. Trapnell C, Hendrickson DG, Sauvageau M, Goff L, Rinn JL, Pachter L. Differential analysis of gene regulation at transcript resolution with RNA-seq Nature Biotech. 2013;31(1):46-53.

48. Anders $\mathrm{S}$, Huber W. Differential expression analysis for sequence count data. Genome Biol. 2010;11(10):R106.

49. Endelman JB. Ridge regression and other kernels for genomic selection with R package rrBLUP. Plant Genome. 2011;4(3):250-5.

\section{Submit your next manuscript to BioMed Central and we will help you at every step:}

- We accept pre-submission inquiries

- Our selector tool helps you to find the most relevant journal

- We provide round the clock customer support

- Convenient online submission

- Thorough peer review

- Inclusion in PubMed and all major indexing services

- Maximum visibility for your research

Submit your manuscript at www.biomedcentral.com/submit
C) Biomed Central 